\title{
Series of International Conferences "Fundamental Problems of High Temperature Superconductivity" and Three Commemorated Events in This Field in the Year 2011
}

\author{
Yurii Kopaev • Vladimir Pudalov
}

Received: 2 May 2013 / Accepted: 8 May 2013 / Published online: 2 June 2013

(C) Springer Science+Business Media New York 2013

FPS' 11 was the 4th Conference in row, organized by the P.N. Lebedev Physical Institute (LPI) of the Russian Academy of Sciences near Russian old city of Zvenigorod. The conference was dedicated to three outstanding events. First, in 2011, the worldwide scientific community celebrated the 100th anniversary of the discovery of superconductivity by H. Kamerlingh-Onnes and collaborators. Second, a quarter of a century ago, J.G. Bednortz and K.A. Müller observed superconductivity at much higher temperatures in cuprate oxides, their discovery opening up a new era in the study of this outstanding phenomenon. And, third, in October 2011, we commemorated the 95th anniversary of Vitalii L. Ginzburg, the 2003 Nobel Prize winner, who has made an outstanding contribution to the theory of this phenomenon; he was one of the pioneers of high temperature superconductivity, inspired and organized comprehensive research at LPI in this area.

Vitaly Ginzburg and HTS. In 1940 Vitalii Ginzburg (VL) attended a speech on superconductivity by Lev Landau (LL), who treated it as the superfluidity of electron liquid in metals. VL was impressed by this work and starting 1943 he engaged in studying superconductivity. No thorough theory of superconductivity existed at that time. From the London theory it follows that the surface energy at the N-S interface is always negative; VL understood that the London theory had to be generalized. To solve this problem, in 1950, Lev Landau and Vitaly Ginzburg elaborated an elegant phenomenological theory which may be viewed as an application of the Landau theory of phase transitions to superconductivity. In this case, a scalar complex function $\Psi$ plays the

Y. Kopaev · V. Pudalov $(\bowtie)$

P.N. Lebedev Physical Institute, Russian Academy of Sciences,

Leninskiy Prospect, 53, Moscow, Russia

e-mail: Pudalov@lebedev.ru role of the order parameter. Therefore, VL preferred to call it the " $\Psi$-theory", though it is more often referred to now as "GLAG" (Ginzburg-Landau-Abrikosov-Gorkov) theory. With the advent of the BCS theory of superconductivity in 1957 , it became clear that the underlying microscopic mechanism is pairing of electrons with opposite momentum and spins. Interestingly, yet in 1952, VL noted that the charged Bose gas would behave like a superconductor, but he did not arrive then at the idea of electron pairing.

The question of radical elevation of the critical temperature was clearly posed for the first time by Little in 1964. VL was very impressed by the newly opened prospective of achieving the superconductivity at much higher temperatures and with a great enthusiasm turned to the problem of high temperature superconductivity in a broad sense, considering various issues. He worked with a group of theorists at LPI, including D. Kirzhnits, E. Maksimov, and others. Their theoretical research is summarized in the monograph "High Temperature Superconductivity" [1] published in 1982, i.e. four years prior to the discovery of HTSC in 1986-1987! The net theoretical result of these studies is as follows: there is no theoretical restriction that would impede achieving superconductivity at essentially higher temperatures, such as e.g. room temperature. VL considered practical achievement of the room temperature superconductivity as a task of the major priority and in 2006 initiated development of largescale experimental research in this field at LPI.

$\mathrm{Fe}$-based HTS (Fe-HTS) pnictides are the most recent "family" of superconductors discovered and their studies were the focus of FPS'11. The exact mechanism that facilitates superconductivity in Fe-HTS is still intriguing. There are several reasons why the Fe-HTS are important to study [2-4]. First, they promise interesting physics that stems from the coexistence of superconductivity and magnetism, and possibly manifest an unusual symmetry of the supercon- 
ducting order parameter. Second, having multi-band electronic structure and strong magneto-elastic coupling, they give hope to revealing and disentangling contributions of the phonon, spin-fluctuations, and electronic glues that are responsible for the superconducting pairing. Below we briefly illustrate these issues.

Finally, Fe-HTS are quite interesting for applications. The very high critical fields, comparable with or superior to those of cuprates, and isotropic critical currents make them potentially promising for electric power and high magnetic field applications. Due to the small Fermi pockets, Fermi velocities, $v_{\mathrm{F}}$, are low, resulting in extremely short coherence length $\xi \sim \hbar v_{\mathrm{F}} / 2 \pi k_{\mathrm{B}} T_{\mathrm{c}} \sim 1-3 \mathrm{~nm}$ and, consequently, very high values of the upper critical field $H_{\mathrm{c} 2}=\phi_{0} / 2 \pi \xi^{2}$ exceeding $100 \mathrm{~T}$ [5-8]. The 122-family Fe-HTS demonstrate high and almost isotropic critical current density, $J_{\mathrm{c}}$, in the range of $10^{5} \div 10^{6} \mathrm{~A} / \mathrm{cm}^{2}$ at $T=4.2 \mathrm{~K}[8,9]$ and relatively low $H_{\mathrm{c} 2}$ anisotropy $\gamma=H_{\mathrm{c} 2}^{(a b)} / H_{\mathrm{c} 2}^{(c)}=1-2[7,8]$. Due to strong intrinsic pinning the critical currents exceed those for the cuprate HTS already in fields above $\sim 15 \mathrm{~T}$.

From the proximity of the AFM and superconducting phases on the phase diagram, it was proposed that pairing in Fe-HTS is mediated by spin-fluctuations [2]. For the twoband superconductor, there is an interesting possibility of the $\mathrm{s} \pm$ pairing symmetry first pointed out in [10] that assumes the sign change of the superconducting order parameter [11, 12]. The indirect band overlap, like in semimetals, makes them susceptible to electronic instability with a wavevector separating the electron and hole pockets. In accordance with the excitonic insulator approach [13, 14], the system is then susceptible either to a spin (charge) density wave or a superconducting instability. The spin dynamics in Fe-HTS is revealed primarily by inelastic neutron scattering (INS) measurements. The resonance in the dynamic spin susceptibility occurs due to its divergence through a sign change of the superconducting order parameter on different parts of the Fermi surface [15]. Remarkably, the resonance observed in INS-experiments coincides well with the larger of two superconducting gaps for various Fe-HTS, measured by point-contact spectroscopy [16].

With regard to conventional phonon coupling, the situation a priori is not favorable since the density of states at the Fermi level is very low and the material does not lend itself to strong electron-phonon coupling [2,3]. On the other hand, several arguments in favor of the phonon coupling come from isotope effect measurements [3], and from ARPES data [3, 17, 18]. The measured gap anisotropy is hard to reconcile with the $\mathrm{s} \pm$ models and spin fluctuations but it fits the s++ models based on orbital fluctuations assisted by phonons $[19,20]$. Also, the $2 \Delta / T_{\mathrm{c}}$ ratios are only slightly enhanced relative to the weak coupling conventional BCS value [16]. The correlation of the Van Hove singularities in the vicinity to the Fermi level (Lifshitz transition) with the onset of superconductivity has been observed in Fe-HTS $[21,22]$. This suggests that the proximity to the electronic topological transition, for one of the multiple FS sheets, favors the superconducting pairing [23, 24].

To summarize, studying interplay of superconductivity and magnetism as well as pairing mechanisms in novel Febased superconductors offers exciting interesting physics. Very instructive and fruitful is to compare properties of these multi-band compounds with those of cuprates. The complexity and multi-band electronic structure of the Fe-HTS promises a good possibility to establish empiric relations between their band structure, magnetic and superconducting properties, and to test various theoretical models.

\section{Final}

The mechanisms of superconductivity and magnetism in FeHTS remain unresolved issues. The true large-scale application of the HTS technology in the ubiquitous surroundings is still emerging and the time of its advent depends critically on the progress in achieving $T_{\mathrm{c}}$ 's as high as up to room temperature. The room temperature superconductivity was a "blue dream" of Vitalii Ginzburg, who inspired and organized comprehensive research in this area, and to whose 95th anniversary the FPS' 11 Conference has been dedicated.

\section{References}

1. Ginzburg, V.L., Kirzhnits, D.A. (eds.): High Temperature Superconductivity. Consultants Bureau, New York (1982)

2. Wilson, J.A.: J. Phys. Condens. Matter 22, 203201 (2010)

3. Sadovskii, M.V.: Phys. Usp. 51, 1201 (2008)

4. Kordyuk, A.A.: Iron-based superconductors: magnetism, superconductivity and electronic structure. Low Temp. Phys./Fiz. Nizk. Temp. 38(9), 1119-1134 (2012)

5. Gurevich, A.: Rep. Prog. Phys. 74, 124501 (2011)

6. Hunte, F., Jaroszynski, J., Gurevich, A., Larbalestier, D.C., Jin, R., Sefat, A.S., McGuire, M.A., Sales, B.C., Christen, D.K., Mandrus, D.: Nature 453, 903 (2008)

7. Yuan, H.Q., Singleton, J., Balakirev, F.F., Baily, S.A., Chen, G.F., Luo, J.L., Wang, N.L.: Nature 457, 565 (2009)

8. Pervakov, K.S., Vlasenko, V.A., Khlybov, E.P., Zaleski, A., Pudalov, V.M., Eltsev, Yu.F.: Supercond. Sci. Technol. 26, 015008 (2012)

9. Fang, L., Jia, Y., Schlueter, J.A., Kayani, A., Xiao, Z.L., Claus, H., Welp, U., Koshelev, A.E., Crabtree, G.W., Kwok, W.-K.: Phys. Rev. B 84, 140504(R) (2011)

10. Mazin, I.I., et al.: Phys. Rev. Lett. 101, 057003 (2008)

11. Hirschfeld, P.J., Korshunov, M.M., Mazin, I.I.: Gap symmetry and structure of Fe-based superconductors. Rep. Prog. Phys. 74, 124508 (2011)

12. Chubukov, A.: Annu. Rev. Condens. Matter Phys. 3, 57 (2012)

13. Keldysh, L.V., Kopaev, Yu.V.: Fiz. Tverd. Tela 6, 2791 (1964). Sov. Phys. Solid State 6, 2219 (1965)

14. Halperin, B.I., Rice, T.M.: Rev. Mod. Phys. 40, 755 (1968)

15. Monthoux, P., Scalapino, D.J.: Phys. Rev. Lett. 72, 1874 (1994)

16. Ponomarev, Y.G., Kuzmichev, S.A., Kuzmicheva, T.E., Mikheev, M.G., Sudakova, M.V., Tchesnokov, S.N., Volkova, O.S., Vasiliev, A.N., Pudalov, V.M., Sadakov, A.V., Usol'tsev, A.S., Wolf, Th., Khlybov, E.P., Kulikova, L.F.: J. Supercond. Nov. Magn. (2013). doi:10.1007/s10948-013-2264-7 in this issue 
17. Kordyuk, A.A., Zabolotnyy, V.B., Evtushinsky, D.V., Kim, T.K., Morozov, I.V., Kulić, M.L., Follath, R., Behr, G., Buechner, B., Borisenko, S.V.: Phys. Rev. B 83, 134513 (2011)

18. Borisenko, S.V., Zabolotnyy, V.B., Kordyuk, A.A., Evtushinsky, D.V., Kim, T.K., Morozov, I.V., Follath, R., Buechner, B.: Symmetry 4, 251 (2012)

19. Onari, S., Kontani, H.: Phys. Rev. Lett. 103, 177001 (2009)

20. Kontani, H., Onari, S.: Phys. Rev. Lett. 104, 157001 (2010)

21. Liu, C., Kondo, T., Fernandes, R.M., Palczewski, A.D., Mun, E.D., Ni, N., Thaler, A.N., Bostwick, A., Rotenberg, E.,
Schmalian, J., Bud'ko, S.L., Canfield, P.C., Kaminski, A.: Nat. Phys. 6, 419 (2010)

22. Liu, C., Kondo, T., Fernandes, R.M., Palczewski, A.D., Mun, E.D., Ni, N., Thaler, A.N., Bostwick, A., Rotenberg, E., Schmalian, J., Bud'ko, S.L., Canfield, P.C., Kaminski, A.: Phys. Rev. B 84, 020509 (2011)

23. Innocenti, D., Poccia, N., Ricci, A., Valletta, A., Caprara, S., Perali, A., Bianconi, A.: Phys. Rev. B 82, 184528 (2010)

24. Innocenti, D., Caprara, S., Poccia, N., Ricci, A., Valletta, A., Bianconi, A.: Supercond. Sci. Technol. 24, 015012 (2011) 